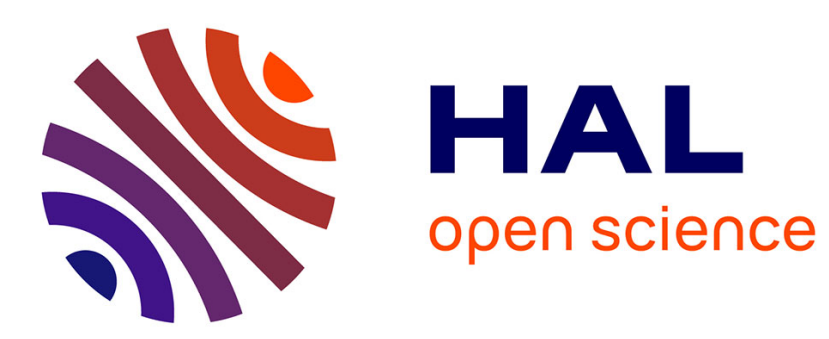

\title{
On the instantaneous formation of cavitation in hydrodynamic lubrication
}

Jesus Ildefonso Diaz, Sébastien Martin

\section{To cite this version:}

Jesus Ildefonso Diaz, Sébastien Martin. On the instantaneous formation of cavitation in hydrodynamic lubrication. 2006. hal-00090870

\section{HAL Id: hal-00090870 https://hal.science/hal-00090870}

Preprint submitted on 4 Sep 2006

HAL is a multi-disciplinary open access archive for the deposit and dissemination of scientific research documents, whether they are published or not. The documents may come from teaching and research institutions in France or abroad, or from public or private research centers.
L'archive ouverte pluridisciplinaire HAL, est destinée au dépôt et à la diffusion de documents scientifiques de niveau recherche, publiés ou non, émanant des établissements d'enseignement et de recherche français ou étrangers, des laboratoires publics ou privés. 


\title{
On the instantaneous formation of cavitation in hydrodynamic lubrication
}

\author{
Jesús Ildefonso Díaz ${ }^{a}$, Sébastien Martin a,b \\ ${ }^{a}$ Dept. de Matemática Aplicada, Fac. de Ciencias Matemáticas, Universidad Complutense Madrid, 28040 Madrid, Spain \\ ${ }^{\mathrm{b}}$ Institut Camille Jordan CNRS-UMR 5208, INSA Lyon, Bât. Léonard de Vinci, F-69621 Villeurbanne Cedex, France.
}

\begin{abstract}
We consider the Elrod-Adams model extending the classical lubrication Reynold equation to the case of the possible presence of a cavitation region. We show that the behaviour of the pressure and saturation depends crucially of the behaviour of the separation $h(t, x, y)$ among the two surfaces. In particular, we exhibit some simple formulations for which we prove (rigorously) that a cavitation region is formed instantaneously (even for initially saturated flows). Some numerical experiences are also given. To cite this article: J.I. Díaz, S. Martin, ...

Résumé

Sur la formation instantanée de la cavitation en lubrification hydrodynamique. Nous considérons le modèle d'Elrod-Adams, qui permet d'étendre l'équation de Reynolds (classiquement utilisée en lubrification) à la prise en compte de la cavitation (formation de bulles de gaz). Nous montrons que le comportement de la presion et de la saturation du lubrifiant dépend, de manière cruciale, du comportement de la distance entre les deux surfaces $h(t, x, y)$. En particulier, nous établissons deux formulations simples pour lesquelles nous établissons (rigoureusement) qu'une zone cavitée se forme instantanément (y compris à partir d'une situation initiale totalement saturée). Des résultats numériques sont également présentés. Pour citer cet article : J.I. Díaz, S. Martin, ...
\end{abstract}

\section{Version française abrégée}

On s'intéresse à un problème à frontière libre issu de la théorie de la lubrification. La modélisation d'un écoulement mince en régime transitoire est généralement basée sur l'équation de Reynolds. Afin de prendre en compte les phénomènes de cavitation (apparition de bulles de gaz dans l'écoulement liquide, à pression constante), on utilise le modèle d'Elrod-Adams, qui introduit une non-linéarité spécifique, par l'intermédiaire d'une inconnue supplémentaire. Ce modèle s'écrit :

$$
\partial_{t}(\theta h)-\operatorname{div}\left(h^{3} \nabla u\right)=v \partial_{x}(\theta h), \quad u \geq 0, \quad \theta \in H(u), \quad \operatorname{sur} Q_{T}=(0, T) \times \Omega .
$$

Email addresses: ji_diaz@mat.ucm.es (Jesús Ildefonso Díaz), sebastien.martin@insa-lyon.fr (Sébastien Martin). 
Ici, $h$ est la hauteur normalisée entre les deux surfaces qui confinent l'écoulement (donnée du problème), $v$ est la vitesse de cisaillement (constante) du dispositif, $u$ est la pression dans le film mince (inconnue du problème), $\theta$ est la saturation locale de la phase liquide et $H$ désigne le graphe de Heaviside.

Bien que ce problème soit bien posé, l'étude de ce modèle, conservatif en termes de débit, présente un enjeu majeur dans la mesure où la naissance des zones dites cavitées (les ensembles tels que $u=0$ ) est un phénomène mal compris. Le but de cette note est de clarifier les conditions d'apparition ou de disparition de la cavitation, à partir de critères géométriques simples.

Théorème 0.1 Supposons qu'il existe $\varepsilon>0$ tel que

$$
D_{t} h=\partial_{t} h+v \partial_{x} h \geq \varepsilon, \operatorname{sur} \overline{Q_{T}} .
$$

Supposons que la condition aux limites suivante est imposée : $u=0$ sur $(0, T) \times \Gamma_{0}$, avec $\Gamma_{0}=\{x \in$ $\left.\partial \Omega, n(x) \cdot e_{x}>0\right\}$ et $\Gamma_{+}=\partial \Omega \backslash \Gamma_{0}$. Supposons de plus $\|u\|_{\infty}$ contrôlé par une certaine constante qui dépend de $\Omega, h$ et de $\varepsilon$ (voir corps du texte). Alors il existe $L \equiv L(h, M, v)>0$ tel que $u(t, x, y)=0$ pour $t \in(0, T]$ et p.p. $(x, y) \in \Omega$ tel que $d\left((x, y), \Gamma_{+}\right) \geq L$. De plus, $\theta(t, x, y)<1$ pour tout $t \in(0, v(x-L)]$ et p.p. $(x, y) \in \Omega$ tel que $d\left((x, y), \Gamma_{+}\right)>L$.

Ce résultat établit l'apparition instantanée de la cavitation sous l'effet de la condition géométrique, ce qui est un résultat radicalement différent de ceux obtenus pour des problèmes paraboliques de type réactiondiffusion pour lesquels il existe un temps de relaxation pour l'apparition de phénomènes similaires. Par ailleurs, ce résultat est local en temps et ne garantit pas la persistance de zones cavitées, ce qui sera illustré numériquement. Une condition suffisante de non-apparition de la cavitation est également établie. Celle-ci peut se révéler fondamentale dans la perspective d'un contrôle local de la cavitation.

Théorème 0.2 Supposons

$$
D_{t} h=\partial_{t} h+v \partial_{x} h \leq 0, \operatorname{sur} \overline{Q_{T}} .
$$

De plus, supposons qu'il n'y a pas de cavitation à l'instant initial. Alors $u>0$ sur $Q_{T}$.

Les résultats sont établis par comparaison avec des sur-solutions et sous-solutions, construites de manière adéquate. Par ailleurs, des tests numériques permettent d'illustrer l'apparition instantanée de la cavitation suivie de sa disparition en temps fini sous l'influence des conditions aux limites.

\section{Introduction}

The Reynolds equation was already proposed in 1886 to describe the behavior of a viscous flow between two close surfaces in relative motion [10]. Nevertheless, the pioneering modelling does not take into account cavitation phenomena: cavitation is defined as the rupture of the continuous film due to the formation of gas bubbles which makes the Reynolds equation no longer valid in the cavitation area. Since then several corrections terms were introduced by different authors. In this paper, we use the Elrod-Adams model [9], introduced in 1975, which assumes that the cavitation region is a liquid-gas mixture giving rise to an additional unknown variable $\theta$ : the liquid saturation in the mixture. If, for the sake of simplicity, we take as 0 the vapor pressure, then a free boundary may be formed separating two different time-spatial zones: the "saturated" region, for which $u>0$ and $\theta=1$ (where the classical Reynolds equation holds) and the "cavitated" region, for which $u=0$ and $0 \leq \theta \leq 1$ (partial lubrication). Thus, $\theta$ describes the local ratio of the liquid phase between the surfaces. The main point about the Elrod-Adams model relies on its physical interest: unlike some other models (such as the variational inequalities model), it is mass-flow preserving and allows to obtain further information in cavitated areas, providing the saturation of the lubricant between the surfaces. This model is widely used in tribology and appears to give satisfactory results with 
respect to mechanical experiments, at least for the stationary version of the model. The dynamic model is also often used, but some questions where not clear, such as, for instance, the formation of cavitation.

The main goal of this note is to point out rigorously how the behavior of the pressure and saturation crucially depends on the behavior of the gap $h(t, x, y)$. In particular, in the best of our knowledge, by first time in the literature, we exhibit some simple formulations for which we can prove that a cavitation region is formed instantaneously (even for initially saturated flows). Some numerical experiments confirm the results and allows to conjecture similar properties under more sophisticated formulations.

\section{A simple mathematical formulation and the main results}

Altough the techniques of this paper can be applied under a larger generality, in order to fix ideas, we shall restrict ourselves to the simple case of a rectangular domain $\Omega=] 0, L_{1}[\times] 0, L_{2}[$; we define the boundaries $\left.\Gamma_{0}=\left\{L_{1}\right\} \times\right] 0, L_{2}\left[\right.$ and $\Gamma_{+}=\partial \Omega \backslash \Gamma_{0}$. Introducing a time $T>0$, we denote $Q_{T}=(0, T) \times \Omega$, $\Sigma_{+}=(0, T) \times \Gamma_{+}, \Sigma_{0}=(0, T) \times \Gamma_{0}$. On the separation funtion $h(t, x, y)$ between both surfaces we assume Assumption 1 (Gap) Let $h \in C^{1}\left(\overline{Q_{T}}\right)$. There exists $\underline{h}, \bar{h}$ and $G$ such that

$$
0<\underline{h} \leq h(t, x, y) \leq \bar{h} \text { and }\left|\nabla h^{3}(t, x, y)\right| \leq G \text { for any }(t, x, y) \in \overline{Q_{T}} \text {. }
$$

Searching a simple formulation, we assume that the flow is moving with the uniform velocity $v \mathbf{e}_{x}$ so that the Elrod-Adams formulation of the hydrodynamic lubrication equation leads to the PDE

$$
\partial_{t}(\theta h)-\operatorname{div}\left(h^{3} \nabla u\right)=-v \partial_{x}(\theta h), \quad u \geq 0, \quad \theta \in H(u)
$$

where $H(u)$ represents the maximal monotone graph of $\mathbb{R}^{2} H(u)=\{0\}$ if $u<0, H(u)=\{1\}$ if $u>0$ and $H(0)=[0,1]$. We must impose

$$
u(0, \cdot)=u_{0}, \theta(0, \cdot)=\theta_{0} \in H\left(u_{0}\right) \text { on } \Omega,
$$

and some of the boundary conditions. To simplify the formulation we assume that

$$
u=0 \text { on } \Sigma_{0} .
$$

Neverthelees, for some of our results, we do not need to prescribe the concrete boundary condition satisfied on $\Sigma_{+}$but merely to assume that there exists $M>0$ such that

$$
u \leq M \text { on } \Sigma_{+} .
$$

Due to this ambiguity, we need to assume something that usually is a (nontrivial) result:

$$
\text { the comparison principle holds. }
$$

We assume the reader familiarized with the notions of super and subsolution. Let us indicate that the conditions (7), for a suitable positive constant $M$, and (8) hold, when we assume some concrete boundary condition on $\Sigma_{+}$(see e.g. [2]). Obviously (7) is satisfied in the special case of the Dirichlet condition

$$
u=M \text { on } \Sigma_{+} \text {. }
$$

Also the comparison principle can be proved by using the techniques of the $\mathrm{L}^{1}$-accretive operators [8] but that a more satisfactory criterium is available by using the technique of doubling variables introduced by S.N. Kruzhkov and then extented by J. Carrillo and other authors (see e.g. [1,2] and references).

Now, let us focus on some qualitative properties of local weak solutions of (4), (5), (6) satisfying (7). Our main contibution is to complement the propagation results obtained in Carrillo, Díaz and Gilardi [6] concerning the special case of $h$ a constant, by point out how the sign of the combination $\partial_{t} h+v \partial_{x} h$ plays a crucial rol in the formation and propagation of a possible unsaturated cavitation region (where 
$u=0$ and $\theta<1)$. We start by considering the more favourable case for the formation and propagation of the cavitation region. The following local result leads to many different global applications.

Theorem 2.1 Assume that there exists $\varepsilon>0$ such that

$$
D_{t} h=\partial_{t} h+v \partial_{x} h \geq \varepsilon, \text { on } \overline{Q_{T}} .
$$

Assume that

and let

$$
M \leq \frac{\varepsilon \min \left(L_{1}, L_{2} / 2\right)^{2}}{4 \bar{h}^{3}+2 G \max \left(L_{1}, L_{2}\right)},
$$

$$
L \equiv L(h, M, v):=\sqrt{\frac{M\left(4 \bar{h}^{3}+2 G \max \left(L_{1}, L_{2}\right)\right.}{\varepsilon}} .
$$

Then $u(t, x, y)=0$ for $t \in(0, T]$ and a.e. $(x, y) \in \Omega$ such that $d\left((x, y), \Gamma_{+}\right) \geq L$. Moreover $\theta(t, x, y)<1$ for any $t \in(0, v(x-L)]$ and a.e. $(x, y) \in \Omega$ such that $d\left((x, y), \Gamma_{+}\right)>L$.

Remark 1 The above result proves how the negative total derivative condition allows the instantaneous formation of a cavitation region: a property which we think holds in more general conditions (see the numerical validation). It means that there is no time relaxation between the initial time and the first time in which the free boundary arises for a given positive initial datum. This behaviour is radically different from the one observed for nonlinear parabolic problems of reaction-diffusion type (see, for instance, the works of Antontsev, Díaz and Shmarev [3] and their references) and, in the best of our knowledge, it is proved here by firt time in the literature.

Our second qualitative property exhibits how the opposite total derivative condition on $h$, ensures, at least, the conservation of the full-saturation of the fluid. Indeed, we have:

Theorem 2.2 Assume the Dirichlet condition (9) on $\Sigma_{+}$and assume that

$$
D_{t} h=\partial_{t} h+v \partial_{x} h \leq 0 \text {, on } \overline{Q_{T}} \text {. }
$$

Assume that $\theta_{0}=1$ a.e in $\Omega$. Then $u>0$ on $Q_{T}$ (and so $\theta(t, x, y)=1$ for any $t \in[0, T]$, a.e $(x, y) \in \Omega$ ).

\section{Sketch of the proofs.}

Proof of Theorem 2.1. We start by introducing a parameter $\omega \in(0,1)$ to be indicated later. In a first step, we construct $\bar{u}(t, x, y)$ as the unique solution of the family of stationary problems ( $t$ being a parameter)

$$
\left\{\begin{array}{l}
-\operatorname{div}\left(h^{3} \nabla \bar{u}\right)+\varepsilon(1-\omega) H(\bar{u}) \ni 0 \quad \text { in } Q_{T}, \\
\bar{u}=0 \quad \text { on } \Sigma_{0}, \quad \bar{u}=M \quad \text { on } \Sigma_{+} .
\end{array}\right.
$$

By adapting the results of Díaz [7] (see Theorems 2.15 and 1.13), we conclude that $\bar{u}(t, x, y)=0$ for any $t \in(0, T)$ and a.e. $(x, y) \in \Omega$ such that $d\left(\left((x, y), \Gamma_{+}\right) \geq L(\omega)\right.$ with

$$
L(\omega):=\sqrt{\frac{M\left(4 \bar{h}+2 G \max \left(L_{1}, L_{2}\right)\right.}{\varepsilon(1-\omega)}} .
$$

Notice that $L(\omega) \searrow L$ as $\omega \searrow 0$ (given by (12) and that, due to the assumption (11), there exists a $\omega \in(0,1)$ for which the set of points $(x, y) \in \Omega$ such that $d\left(\left((x, y), \Gamma_{+}\right) \geq L(\omega)\right.$ is not empty. Now, by using $(10)$ it is easy to check that $(\bar{u}, 1)$ is a supersolution since, for this $\omega$, the PDE can be decomposed as

$$
\left[h D_{t} \theta+\omega \theta D_{t} h\right]+\left[-\operatorname{div}\left(h^{3} \nabla u\right)+(1-\omega) \theta D_{t} h\right]=0 .
$$


Then, by the comparison principle (8) we conclude that $u(t, x, y)=0$ for $t \in(0, T]$ and a.e. $(x, y) \in \Omega$ such that $d\left(\left((x, y), \Gamma_{+}\right) \geq L(\omega)\right.$. In a second step, given a point $\left(x^{0}, y^{0}\right) \in \Omega$ such that $d\left(\left(\left(x^{0}, y^{0}\right), \Gamma_{+}\right) \geq L(\omega)\right.$, we shall define a function $\bar{\theta}(t, x, y)$ with $\bar{\theta} \in H(\bar{u})$, for $t$ small enough and a.e. $(x, y) \in \Omega$ such $x \leq x^{0}$. This function $\bar{\theta}$ is constructed as given by 1 for a.e. $(x, y) \in] 0, x^{0}[\times] 0, L_{2}\left[\right.$, and for $t \in\left[0, v\left(x^{0}-L\right)\right]$, except on the set of points $R_{\left(x^{0}, y^{0}\right)}^{\delta}:=\{(x, y) \in] 0, x^{0}[\times] y^{0}-\delta, y^{0}+\delta\left[\right.$ such that $d\left(\left((x, y), \Gamma_{+}\right) \geq L(\omega)\right\}$ (with $2 \delta<d\left(\left(\left(x^{0}, y^{0}\right), \Gamma_{+}\right)-L(\omega)\right)$, where we take

$$
\begin{cases}\bar{\theta}(t, x, y)=1 & \text { if }(x, y) \in R_{\left(x^{0}, y^{0}\right)}^{\delta} \text { and } t+v\left(x^{0}-L\right) \leq 0 \\ \bar{\theta}(t, x, y)=\exp \left(-\frac{\omega \varepsilon}{\bar{h}}(t+v(x-L(h, M)))\right) & \text { if }(x, y) \in R_{\left(x^{0}, y^{0}\right)}^{\delta} \text { and } t+v\left(x^{0}-L\right)>0 .\end{cases}
$$

Notice that $\Theta(s)=\exp (-(\varepsilon \omega / \bar{h}) s)$ verifies that $\Theta(0)=1$ and $\Theta^{\prime}(s)+(\varepsilon \omega / \bar{h}) \Theta(s)=0$ for $s>0$. Now, since $t \in\left[0, v\left(x^{0}-L\right)\right]$ (thanks also to the first step), we get that $\bar{\theta} \in H(\bar{u})$ on the spacial set $] 0, x^{0}[\times] 0, L_{2}[$ and it is not difficult to check that $(\bar{u}, \bar{\theta})$ is, there, a supersolution (by using $(10)$ ). By the comparison principle, we get that $\theta\left(t, x^{0}, y^{0}\right) \leq \Theta(t)<1$ for any $t \in\left(0, v\left(x^{0}-L\right)\right]$ which ends the proof.

Proof of Theorem 2.2. Let $\underline{u}$ be the unique solution of the stationary problems ( $t$ being a parameter)

$$
\begin{cases}-\operatorname{div}\left(h^{3} \nabla \underline{u}\right)=0 & \text { in } Q_{T}, \\ \underline{u}=0 \quad \text { on } \Sigma_{0}, & \underline{u}=M \quad \text { on } \Sigma_{+} .\end{cases}
$$

By the strong maximum principle, $\underline{u}>0$ on $Q_{T}$ and by assumption $(13),(\underline{u}, 1)$ is obviously a subsolution.

\section{Some numerical results}

In this section, we choose $\Omega=] 0,2 \pi[\times] 0,1[$. The gap is given by $h(t, x, y)=(1+0.5 \cos (x)) f(t)$. The initial condition is $u(0, x, y):=u_{0}(x, y)=0.5(1-x / 2 \pi)$ and $\theta(0, x, y)=1$ and the boundary condition is $u=\gamma\left(u_{0}\right)$ on $(0, T) \times \partial \Omega, \gamma$ being the trace operator. Numerical results are obtained with a method by Bayada, Chambat and Vázquez [4], adapted to evolutive problems. Numerical data are the following ones: the mesh parameters are $\Delta x_{1}=2 \pi / 100, \Delta x_{2}=1 / 100$, the time step $\Delta t=v \Delta x_{1}($ with $v=1)$.

Theorem 2.1 is illustrated by the numerical test: taking $f(t)=e^{+1.1 t}$, Fig.1-2 show that cavitation immediately appears in a significant way between $t_{0}$ and $t_{1}$, due to the constraint (10), and then tends to disappear due to the influence of the boundary pressure. From a numerical point of view, a refinement of the time step would lead to the same observations, meaning that there is no time relaxation between the birth of cavitation and the time when (10) is satisfied.

Theorem 2.2 may be illustrated in the following way: taking $f(t)=e^{-1.1 t}$ in the definition of the gap and starting from a full-satured configuration, as (13) is satisfied, then we would observe (figures have been omitted for convenience) that no cavitation appears, even in the spatial diverging parts of the device.

Actually, even if (10) or (13) is not satisfied in the whole domain, the local behaviour of the solution still highly depends on the constraint at a local level. This may be also illustrated when taking tha classical configuration $f(t)=1$ (i.e. stationary gap), for which it can be observed that cavitation only appears in the spatial diverging part while no cavitation appear in the spatial converging parts of the device. 

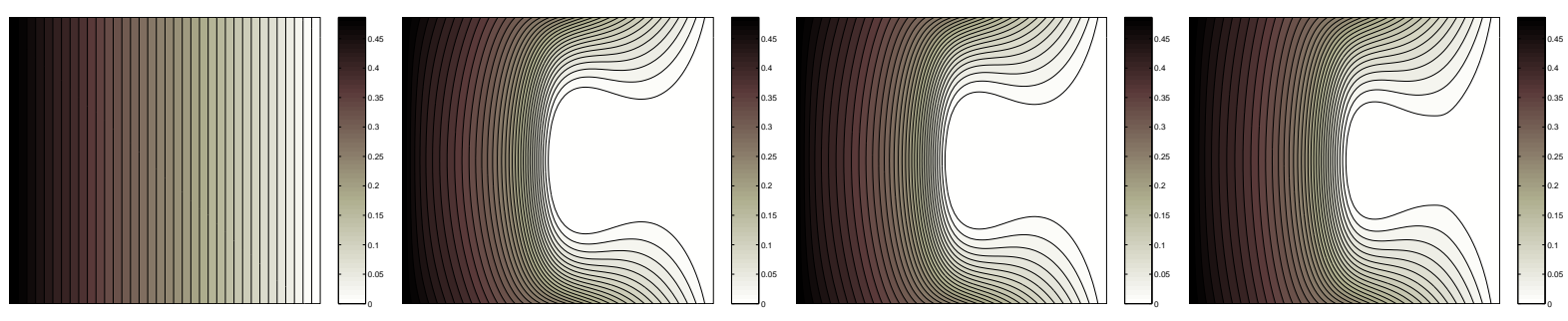

Figure 1. Pressure at times $t_{0}, t_{1}, t_{2}, t_{4}\left(\right.$ with $\left.t_{i}=i \Delta t\right)$
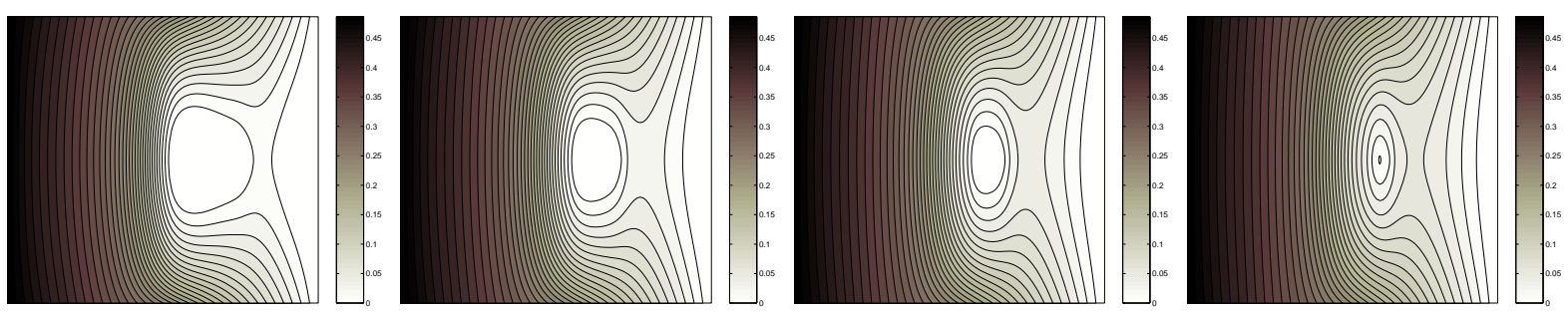

Figure 2. Pressure at times $t_{5}, t_{7}, t_{8}, t_{9}\left(\right.$ with $\left.t_{i}=i \Delta t\right)$

\section{Acknowledgements}

The authors are grateful to S. Alvarez and C. Vázquez for fruitful discussions. This work was realised during the posdoctoral visit in Madrid of S. Martin, supported by the RTN HPRN-CT-2002-00274 (EU). The research of J. I. Díaz was supported by the project MTM-2005-03463 of the DGISGPI (Spain).

\section{References}

[1] S.J. Alvarez, J. Carrillo, A free boundary problem in theory of lubrication. Comm. Partial Differential Equations, 19(11-12):1743-1761, 1994.

[2] S.J. Alvarez, R. Oujja, On the uniqueness of the solution of an evolution free boundary problem in theory of lubrication. Nonlinear Anal., 54(5):845-872, 2003.

[3] S.N. Antontsev, J.I. Díaz, S.I. Shmarev, Energy methods for free boundary problems. Applications to nonlinear PDEs and Fluid Mechanics, Series Progress in Nonlinear Differential Equations and Their Applications (48), Birkhäuser Boston, 2002.

[4] G. Bayada, M. Chambat, C. Vázquez, Characteristics method for the formulation and computation of a free boundary cavitation problem. J. Comput. Appl. Math., 98(2):191-212, 1998.

[5] G. Capriz, G. Cimatti, Partial lubrication of full cylindrical bearings. ASME J. Lubrication Technol., 105:84-89, 1983.

[6] J. Carrillo, J. I. Díaz, G. Gilardi, The propagation of the free boundary of the solution of the dam problem and related problems. Applicable Analysis, 4:255-276, 1993

[7] J. I. Díaz, Nonlinear Partial Differential Equations and Free Boundaries. Elliptic equations. Research Notes in Mathematics, I(106), Pitman, Londres, 1985.

[8] J. I. Díaz, On the mathematical analysis of transient cavitation problems in Hydrodynamics Lubrification. In, Mathematical Modelling Lubrification (G. Bayada, M. Chambat and J. Durany eds.) Univ. de Vigo, 37-49, 1991.

[9] H.G. Elrod, M.L. Adams A computer program for cavitation. Cavitation and related phenomena in lubrication Proceedings - Mech. Eng. Publ. ltd, 37-42, 1975.

[10] O. Reynolds, On the theory of lubrication and its application to Mr Beauchamp tower's experiments, including an experimental determination of the viscosity of olive oil. Phil. Trans. Roy. Soc., A(117):157-234, 1886. 